BRX-TH-6289

CALT-TH-2015-003

\title{
The legacy of ADM
}

\author{
S. Deser \\ Walter Burke Institute for Theoretical Physics, \\ Caltech, Pasadena, California 91125, USA \\ Physics Department, Brandeis University, \\ Waltham, Massachusetts 02454, USA
}

\begin{abstract}
This tribute to the memory of my old friend and collaborator, Richard Arnowitt, focuses on the history, results and physical significance of the Arnowitt-Deser-Misner (ADM) formulation of General Relativity, starting from its birth in 1958-9 through its completion, in a series of over a dozen papers, in 1962-3. A few of its later applications are also mentioned.
\end{abstract}




\section{PREHISTORY}

Dick and I came to Harvard's Physics graduate school essentially simultaneously, although he had already earned an MA from RPI. Our paths were parallel throughout Harvard's course system for the first two years and when we both began theoretical research under our mentor, Julian Schwinger, then an almost unapproachable idol. Those were the years right after the wave of wartime students' influx (Julian alone producing many Nobel laureates, besides himself), and marked the end of the great Quantum Electrodynamics (QED) postwar era.

We each had different postdoc trajectories, but in Fall '58 I found myself back at Harvard as Schwinger's assistant, at a time when everyone was trying to find the next wave. Although I worked on the then fashionable dispersion theory, both Dick and I maintained our love for Quantum Field Theory. Indeed, so did Julian, who began to (re-)think about higher spins, including spin 2 - the basis of General Relativity (GR), which he of course had learned as a child. At that time, GR was an almost forgotten domain, mostly devoted to the botany of exact solutions and other geometrical practices, and frowned on as an occupation for young theoreticians by that era's authorities. [What a difference a few decades makes!] Independently of Schwinger, Dick and I began to apply our knowledge of QED to spin 2 as well, and in that year 58-59, had completed the canonical analysis of the easy part

- linearized GR. It goes without saying that, for us quantum dwellers, the aim was to quantize the theory. [Of course we knew that quantizing the linear limit would be trivial as indeed it was, having been done in the early thirties by Bronstein and Rosenfeld- and that perturbative GR was non-renormalizable, by the already standard old Fermi theory argument using the dimensionality of Newton's constant.]

During that first period, we were summoned by Wheeler, who had heard of, and wanted to be briefed on, our ideas. As we entered his office - which was equipped with a huge tape recorder as well as his notorious bound notebooks - he asked whether he could bring in his student who might have some relevant results for our quest. Being seasoned postdocs, we did not expect much from a mere student, but agreed. After our exposition, Charlie Misner told us of his new formulation of the GR action, and it was suggested we join forces. We were smart enough to realize the benefits and rapidly agreed to do so. Thus was ADM born - and none of us ever looked back. 


\section{ADM: THE HISTORY}

Between 1958-9 and 1963, the three of us collaborated, sometimes in the same spot(s) they were many - by mail, and (rarely) by phone in those prehistoric days. We attended the historic Royaumont Conference in France,the third in the famous series (the traditional numbering is \#0 in Bern in 1955, which only I attended, as an ignorant tourist, \#1 was in Chapel Hill in 1957 where Charlie \& I were present). In Royaumont, we discovered that Dirac was on the same track - but also that our approaches were almost orthogonal, and that we were ahead in results: This was fortunate, because to compete with Dirac would otherwise be enough to discourage anyone! The whole subject was indeed slowly waking from its hibernation from the early twenties: for example, even that first dedicated, 1955 Bern, conference had but 80 participants, of whom far fewer were pros. Still, by the late 50s there were a number of active, if small, groups of younger people working in London, Syracuse, Texas, UNC, Hamburg, Warsaw, Princeton and the USSR. The Warsaw conference of 1962 also included Dirac, as well as Feynman - who mainly (co-) contributed the formal need for ghosts in covariant quantization of nonabelian gauge fields.

The most intense, initial - and productive - 3-way stretch came in the Summer of '59, when we all met on a Danish island and worked on the floor in a Kindergarten, now alas gone, on knee-high blackboards. It was there that we found the basic translation of the geometrical meaning of GR into modern Field Theoretical language. From that realization, all the pieces fell into place - the many results we obtained in our many subsequent papers just kept coming! By 1963,we had more or less found all that was worth doing to set up the physics, and our collaboration - but not our friendship - naturally dissolved on geographic grounds.

\section{ADM - THE PHYSICS}

Since this is intended for a nonspecialized readership, I will only outline the basics of the ADM version of GR; details and extensions may be found in our various papers; the entire story, including individual references, is contained in our major 1962 review paper, now

available online [1]. I emphasize that our formulation is entirely equivalent to geometrical GR, but couched in the then newly developed language of gauge theories. The key equation 
from which many results flow is the Einstein-Hilbert action (the densities also agree up to a total divergence) in $3+1$ [or in any $(D-1)+1]$, in canonical, Hamiltonian $-L=p \dot{q}-H(p, q)$ - first order form and in Planck units,

$$
\begin{aligned}
I & =\int d^{4} x\left[\pi^{i j} \dot{g}_{i j}-N_{\mu} R^{\mu}(\pi, g)\right] \\
R^{0} & ={\sqrt{ }{ }^{3} g}^{3} R+\left(\pi^{2} / 2-\pi^{i j} \pi_{i j}\right) / \sqrt{{ }^{3} g} \\
R^{i} & =-2 D_{j} \pi^{i j} .
\end{aligned}
$$

Here the six conjugate pairs $\left(\pi^{i j}, g_{i j}\right)$ are of course to be varied independently and all operations such as index-shifting and covariant differentiation $D_{i}$ use the 3-metric $g_{i j}$ and its inverse $g^{i j}$. All variables are combinations of the metric and second fundamental form:

$$
\begin{aligned}
& \pi^{i j} \equiv \sqrt{-g} g^{i p} g^{j q}\left(\Gamma_{p q}^{0}-g_{p q} \Gamma_{r s}^{0} g^{r s}\right) \\
& N_{0} \equiv N \equiv\left(-g_{00}\right)^{-1 / 2}, N_{i} \equiv g_{0 i} .
\end{aligned}
$$

So $\pi^{i j}$ is essentially the time derivative of the spatial metric $g_{i j}$, as befits a canonical momentum variable, while the Lagrange multipliers $N_{\mu}$ in (3.1) are collectively the set $\left\{g_{0 \mu}\right\}$, nowadays also called the shift $\left(N_{i}\right)$ and lapse $(N)$. The Hamiltonian action (3.1) is both very familiar (as $p \dot{q}-H$ ) and very alien: varying the $N_{\mu}$ yields the four constraints $R^{\mu}=0$ - constraints because they do not involve time derivatives - leaving a vanishing Hamiltonian: now $L=p \dot{q}-0$ !

The resolution of this paradox is key to understanding how geometry, including coordinate invariance, transmutes into "mechanics". What we realized in that Kindergarden was that this phenomenon was already - if artificially - constructed long ago by Jacobi - who showed that the standard action principle for a dynamical system could be parametrized to elevate the number of degrees of freedom by one, and remove the Hamiltonian altogether, replacing it by a constraint on the that new - fake - excitation:

$$
\begin{aligned}
I & =\int L d \tau \\
& =\int\left(\left[\sum_{a=1}^{N} p^{a} d q_{a}+P d Q\right]-N[P+H(p, q)] d \tau\right) \\
& =\int\left(\sum_{a=1}^{N+1} p^{a} d q_{a}-N R(P ; p, q) d \tau\right) .
\end{aligned}
$$


Solving the $R$-constraint for $P$ and choosing $Q$ to be the time recovers the original action. Note the "general covariance" of the last expression under choice of time $\tau$, and its exact ADM form (3.1). So GR is what we called an "already parametrized" theory - with no underlying "normal" action $I=\int d t[p \dot{q}-H(p, q)]$ and time $t$ to take refuge in! There are four constraints, simply because we have a field theory with four coordinates instead of just time. Each constraint removes one degree of freedom (in this first order form), leaving only two of the original $6\left(\pi^{i j}, g_{i j}\right)$ pairs. Concretely, we may take these to be the two transverse-traceless (TT) modes (just two because of the 4 conditions $\partial_{j} \pi^{i j}=0=\pi_{i}^{i}$, etc.). [More generally, they are the equivalents of the two transverse photon excitations $\left(E^{i T}, A_{i}^{T}\right)$ - and indeed of the two helicity $\pm s$ modes of any massless, spin $s>0$ excitations, in $D=4$.] In the weak field limit, they describe the - abelian gauge-invariant - helicity \pm 2 gravitons. We are separately free to decide on what to use as the coordinates $x^{\mu}$, including time - this is coordinate invariance of course - with the corresponding, conjugate, choice of Hamiltonian or rather 4-momentum $P_{\mu}$. [This gauge freedom comes with all sorts of problems of principle regarding gauges that differ by functions of the two "true" excitations, especially in any formal quantization attempt. We discussed these troubling issues in some detail, but they keep being rediscovered.] The physical guide to proper coordinate choices, apart from more exotic questions for spaces with weird topology, is of course the asymptotic - at spatial infinity (rather than null infinity, more suited to non- "3+1" approaches) weak field regime, that is, the usual choice of boundary conditions in field theories, though here there can also exist closed spaces with no infinity - and hence no notion of energy either, as we will see. [Parenthetically, a cosmological constant is easily incorporated, since $\sqrt{-g}=N \sqrt{{ }^{3} g}$, by just adding $\sqrt{{ }^{3} g}$ to $R^{0}$ in (3.1) and altering asymptotic states from flat to $(\mathrm{A}) \mathrm{dS}$ spaces.

Gauge invariance means local quantities such as the gravitational field's stress-tensor are meaningless - but global ones such as $P_{\mu}$ are physical - in particular, energy is well-defined, and is in fact even simply expressed as a surface integral over energy flux at spatial infinity, just like flux of longitudinal electric field counts the total charge $Q$ in Maxwell. This socalled "ADM" energy or mass plays an essential role in every aspect of GR, and indeed in Supergravity (SUGRA), GR's modern successor [2]. In its simplest terms, $E$ is defined from the $R^{0}$ constraint of (3.1), as the conjugate to the simplest asymptotic time choice, best seen by expanding $R^{0}$ into its unique linear term - the linear part of the 3-curvature 
${ }^{3} R \sim \nabla^{2} g^{T}$, where $g^{T}$ is a particular component of $g_{i j}$ in its orthogonal expansion that extends a vector's decomposition into transverse (divergence-less) and longitudinal (curlfree) parts - plus the nonlinear remainder. Then the spatial integral of the Poisson equation $\nabla^{2} g^{T}=-R^{0}$ (nonlinear) is the total energy. [Solutions that do not decay sufficiently rapidly at spatial infinity, or, at the other extreme, closed spaces, do not - and should not - have well defined $E$.] These simple arguments can be formalized in terms of asymptotic Killing vectors, obeying $D_{(i} K_{j)}=0$; indeed this must - and can - be done explicitly to define energy also in the presence of the cosmological term [3] and indeed for arbitrary covariant extensions of GR [4], such as $R+R^{2}$ models. It also has a counterpart in the need for asymptotic Killing "color" vectors to define the total, non-abelian, charge in Yang- Mills models [5] that - unlike Maxwell - are "charged", just as the gravitational field's "charge" is energy.

Establishing $E$-positivity was one of the longest-standing challenges in the field, to which ADM contributed only some special cases; indeed it was not proved until much later. On the other hand, positivity of (necessarily quantum) SUGRA energy, which includes that of GR in the classical limit, was easily established [6] soon after SUGRA itself, basically because that theory is the "Dirac square root" of GR - the very words "square root" almost embody it.

Many other uses of the ADM formulation were explored - the wave zone and gravitational waves could be defined, in analogy with the near and far zones of electrodynamics - this being one major example; to this very day sophisticated numerical studies of radiation use the ADM methods that are especially suited to time evolution of the gravitons' excitation modes as well as to their creation and absorption.

Another deep problem is that of self-energy of massive neutral or charged particles, and more generally the effective particle-particle post-Newtonian interactions. The latter subject has also been pushed to high analytic and numerical order, using the ADM effective matter coupling results; this industry is now at high- $n$ post $^{n}$-Newtonian level, although of course, beyond a certain order, inclusion of radiative effects is unavoidable. Indeed it is a beautiful (and a priori amazing) result that all matter systems couple to gravity in exactly such a way as to keep the sacred form

$$
L\left(\operatorname{matt} ; g_{\mu \nu}\right)=\Sigma_{\text {matt }} p \dot{q}-N_{\mu} R^{\mu}\left(p, q ; g_{i j}, \pi^{i j}\right)
$$


That is, the coupled theory maintains the hallmark of "already parametrization" - or general covariance - in particular the $N R$ form, with $R$ independent of $N_{\mu}$ - when the conjugate matter pairs $(p, q)$ are properly chosen. This general property was later established in a series of long technical papers [7].

Coming back to the self-energy problem, it had long [8] been speculated that GR might dampen self-energy divergences of matter. One amusing indication is the following argument showing that a distribution of bare mass $m_{0}$ had vanishing ADM mass $m$ in the point $(R \rightarrow 0)$ limit, and further that a charged one's limiting mass was proportional to its charge, $m \rightarrow|e|$. It is in fact quite intuitive: for the neutral $m_{0}$, of size $R$, the "Newtonian" energy is of course

$$
E(N)=m_{0}-G m_{0}^{2} / 2 R \rightarrow-\infty
$$

since the self-interaction only involves the bare, mechanical mass, whereas in GR ALL mass $*_{\text {self*-gravitates, so }}$

$$
E(G R)=m_{0}-G m^{2} / R \rightarrow 0
$$

The limit is obtained by solving the quadratic equation (3.11) for $m\left(m_{0}, R\right)$. Physically, this means that the total mass $m$ diminishes from its dilute value $m_{0}$ at $R=\infty$. Once it reaches 0 , however, the process stops $-m$ cannot go negative, there being no mass left to gravitate!

The above amazing - perhaps counterintuitive - result even extends to a charged massive distribution: the effect of the Coulomb interaction, namely adding the extra term $+e^{2} / 2 R$ in (3.10) or (3.11), is as follows: in the Newtonian limit, one gets $E(N) \rightarrow \pm \infty$ depending on the ratio of $e / m_{0}$. Instead, the GR limit is finite:

$$
E(G R) \rightarrow \sqrt{e^{2} / G}
$$

These elementary arguments are borne out by solving the corresponding constraint equations in detail. To be sure, classical finiteness does not imply quantum finiteness, although there has been some quantum corroboration [9] of our above entirely nonperturbative effect. Indeed it is known that in perturbative (our only current general approach) quantum gravity, even low loop orders $L$ are divergent for GR alone $(L=2)$ or coupled to matter of any spin, massive or massless ( $L=1$, except $L=3$ for $N=1$ SUGRA) [10]. The only remaining hope is the very special system of maximal, unbroken $-N=8$ - SUGRA, which is still standing - finite - to high, 7, loop order [11]. 
I will not enter into the immense literature on ADM applications to many concrete problems, ranging from cosmology - there, amongst others, to the dark energy, dark matter and cosmic acceleration problems - to astrophysics, in manifold ways, especially those involving gravitational radiation and matter interactions - to gravitational model building, including extensions of GR and SUGRA [12] - to numerical integration methods, to quantization. I must content myself with this, telegraphic and incomplete, list of topics touched by ADM ideas - essentially all domains of GR and its generalizations. Interested readers will easily encounter specific examples, some even speaking ADM without knowing it.

\section{SUMMARY}

This tribute to my deceased long-term collaborator and friend, Dick Arnowitt, gives an extremely compressed, and incomplete, view of the role played by ADM in all areas of gravitational research, from the conceptual to the numerical. I refer the reader to the original works, catalogued in [1], and to the vast, and ongoing, related literature. ADM was meant to bring GR from 1915 into the late twentieth Century - expressing it in the language of modern field and gauge theories - hoping to clarify its relation to the other three fundamental forces on the one hand, and to provide, in its own domain, a bridge to numerical and analytical applications on the other. In these tasks it has reasonably succeeded. Our only regret is that Dick will not share the forthcoming Einstein Medal for ADM in this, GR's Centennial year.

\section{Acknowledgments}

S.D. was supported in part by NSF and DOE grants PHY-1266107 and \#de-sc0011632.

[1] Richard L. Arnowitt, Stanley Deser, Charles W. Misner, "The Dynamics of General Relativity", in Gravitation, a Modern Introduction, L. Witten Ed. (Wiley, NY, 1962), reprinted in Gen. Rel. Grav. 40 (2008) 1997 and gr-qc/0405109. 
[2] "Consistent Supergravity", S. Deser and B. Zumino (CERN), Phys. Lett. B62 (1976) 335; "Progress Toward a Theory of Supergravity", D. Z. Freedman, P. van Nieuwenhuizen, S. Ferrara, Phys. Rev. D13 (1976) 3214.

[3] "Stability of Gravity with a Cosmological Constant", L.F. Abbott and S. Deser, Nucl. Phys. B195 (1982) 76.

[4] "Energy in generic higher curvature gravity theories", S. Deser, B. Tekin, Phys. Rev. D67 (2003) 084009, hep-th/0212292; "New energy definition for higher curvature gravities", Phys. Rev. D75 (2007) 084032; gr-qc/ 0701140.

[5] "Charge Definition in Nonabelian Gauge Theories", L. F. Abbott, S. Deser, Phys. Lett. B116 (1982) 259.

[6] "Supergravity Has Positive Energy", S. Deser, C. Teitelboim, Phys. Rev. Lett. 39 (1977) 249.

[7] "Geometrodynamics with tensor sources", Karel Kuchar, J. Math. Phys. 18, 1589 (1977).

[8] "General Relativity and the Divergence Problem in Quantum Field Theory", S. Deser, Rev. Mod. Phys. 29, 417 (1957) and references therein.

[9] "Generalizing the ADM Computation to Quantum Field Theory", P. J. Mora, N. C. Tsamis, R. P. Woodard, Class. Quant. Grav. 29 (2012) 025001, grqc/ 1108.4367; "Particles as bound states in their own potentials", R. P. Woodard, in Miami Beach 1997, Physics of Mass 197, gr-qc/9803096.

[10] "One Loop Divergences of the Einstein Yang-Mills System", S. Deser, H.-S.Tsao, P. van Nieuwenhuizen, Phys. Rev. D10 (1974) 3337; "Renormalizability Properties of Supergravity", S. Deser, J. H. Kay, K. S. Stelle, Phys. Rev. Lett. 38 (1977) 527.

[11] "Amplitudes and Ultraviolet Behavior of $\mathrm{N}=8$ Supergravity", Z. Bern, J. J. Carrasco, L. J. Dixon, H. Johansson, R. Roiban, Fortsch. Phys. 59 (2011) 561; hep-th 1103.1848, and ongoing publications.

[12] "Hamiltonian Formulation of Supergravity", S. Deser, J. H. Kay, K. S. Stelle, Phys. Rev. D16 (1977) 2448. 